\title{
Evolution of embryo implantation was enabled by the origin of decidual cells in eutherian mammals
}

\author{
Arun R. Chavan ${ }^{1,2, *}$, Oliver W. Griffith ${ }^{1,2}$, Daniel Stadtmauer ${ }^{1,2}$, Jamie Maziarz ${ }^{1,2}$, Mihaela \\ Pavlicev $^{3}$, Ruth Fishman ${ }^{4}$, Lee Koren ${ }^{4}$, Roberto Romero ${ }^{6,7}$, Günter P. Wagner ${ }^{1,2,5,6, *}$ \\ ${ }^{1}$ Department of Ecology and Evolutionary Biology, Yale University, New Haven, CT, USA \\ ${ }^{2}$ Yale Systems Biology Institute, Yale University, West Haven, CT, USA \\ ${ }^{3}$ Cincinnati Children's Hospital, Cincinnati, OH, USA \\ ${ }^{4}$ The Mina and Everard Goodman Faculty of Life Sciences, Bar-Ilan University, Ramat-Gan, \\ Israel \\ ${ }^{5}$ Department of Obstetrics, Gynecology, and Reproductive Science, Yale Medical School, New \\ Haven, CT, USA \\ ${ }^{6}$ Department of Obstetrics and Gynecology, Wayne State University, Detroit, MI, USA \\ ${ }^{7}$ Perinatology Research Branch, Division of Obstetrics and Maternal-Fetal Medicine, Division \\ of Intramural Research, Eunice Kennedy Shriver National Institute of Child Health and Human \\ Development, National Institutes of Health, Detroit, MI, USA \\ * Correspondence to: arun.chavan@yale.edu and gunter.wagner@yale.edu
}




\section{Abstract}

2 Embryo implantation is the first step in the establishment of pregnancy in eutherian (Placental)

3 mammals. Although viviparity evolved prior to the common ancestor of marsupials and

4 eutherian mammals (therian ancestor), implantation is unique to eutherians. The ancestral therian

5 pregnancy likely involved a short phase of attachment between the fetal and maternal tissues

6 followed by parturition rather than implantation, similar to the mode of pregnancy found in

7 marsupials such as the opossum. Embryo implantation in eutherian mammals as well as embryo

8 attachment in opossum, induce a homologous inflammatory response in the uterus. Here, we

9 elucidate the evolutionary mechanism by which the ancestral inflammatory fetal-maternal

10 attachment was transformed into the process of implantation. We performed a comparative

11 transcriptomic and immunohistochemical study of the gravid and non-gravid uteri of two

12 eutherian mammals, armadillo (Dasypus novemcinctus) and hyrax (Procavia capensis); a

13 marsupial outgroup, opossum (Monodelphis domestica); and compared it to previously published

14 data on rabbit (Oryctolagus cuniculus). This taxon sampling allows inference of the eutherian

15 ancestral state. Our results show that in the eutherian lineage, the ancestral inflammatory

16 response was domesticated by suppressing a detrimental component viz. signaling by the

17 cytokine IL17A, while retaining components that are beneficial to placentation, viz.

18 angiogenesis, vascular permeability, remodeling of extracellular matrix. IL17A mediates

19 recruitment of neutrophils to inflamed mucosal tissues, which, if unchecked, can damage the

20 uterus as well as the embryo and lead to expulsion of the fetus. We hypothesized that the uterine

21 decidual stromal cells, which evolved coincidentally with embryo implantation, evolved, in part,

22 to prevent IL17A-mediated neutrophil infiltration. We tested a prediction of this hypothesis in

23 vitro, and showed that decidual stromal cells can suppress differentiation of human naïve $\mathrm{T}$ cells

24 into IL17A-producing Th17 cells. Together, these results provide a mechanistic understanding of

25 early stages of the evolution of the eutherian mode of pregnancy, and also identify a potentially

26 ancestral function of an evolutionary novelty, the decidual stromal cell-type. 


\section{Introduction}

Embryo implantation is the process by which the blastocyst establishes a sustained fetal-maternal interface for the maintenance of pregnancy. It begins with apposition of the blastocyst to endometrial luminal epithelium, followed by its attachment via molecular interactions, and, in many eutherian species, invasion of the endometrium to establish a direct contact with the endometrial connective tissue and vasculature (Mossman 1937, Enders and Schlafke 1969, Schlafke and Enders 1975, Ashary, Tiwari et al. 2018). Implantation is one of the most critical steps in the establishment of a successful pregnancy, but it only occurs in eutherian mammals (also known as Placental mammals). Mammalian viviparity originated before the common ancestor of eutherian mammals and marsupials, i.e. in the stem lineage of therian mammals. However, marsupial and eutherian pregnancies are different in many fundamental ways, including embryo implantation.

Marsupial pregnancy is very short - in most cases shorter than the ovarian cycle (Renfree 1994, McAllan 2011). For most of the duration of marsupial pregnancy, the embryo is present inside of an eggshell (Selwood 2000, Griffith, Chavan et al. 2017) that precludes a direct physical contact between the fetal and maternal tissues. Towards the end of the pregnancy, the eggshell breaks down and the fetal membranes attach to the uterine luminal epithelium. The phase of attachment lasts a short fraction of the length of gestation, and is soon followed by the birth of highly altricial neonates. For instance, pregnancy in the South American marsupial, the grey short-tailed opossum (Monodelphis domestica), lasts 14.5 days. Embryo attachment begins approximately on the $12^{\text {th }}$ day post-copulation $(\mathrm{dpc})$ and induces an acute inflammatory response in the uterus (Griffith, Chavan et al. 2017). This inflammation is presumably why embryo attachment precipitates parturition rather than implantation (Chavan, Griffith et al. 2017, Hansen, Faber et al. 2017).

Puzzlingly, in many eutherian mammals such as human, mouse, pig, and sheep, embryo implantation also shows signs of an inflammatory reaction; some of these inflammatory processes are in fact necessary and beneficial for a successful implantation (Keys, King et al. 1986, Barash, Dekel et al. 2003, Waclawik and Ziecik 2007, Plaks, Birnberg et al. 2008, Mor, Cardenas et al. 2011, Dekel, Gnainsky et al. 2014, Robertson and Moldenhauer 2014, Chavan, Griffith et al. 2017, Whyte, Meyer et al. 2017). Resemblance of the physiological process of implantation to an inflammatory reaction appears paradoxical at first because inflammation in later stages of pregnancy leads to the termination of pregnancy. However, analysis of the evolutionary history of embryo implantation suggests that this resemblance is due to the evolutionary roots of implantation in an inflammatory response to embryo attachment (Finn 1986, Griffith, Chavan et al. 2017). Griffith and colleagues (Griffith, Chavan et al. 2017, Griffith, Chavan et al. 2018) argued, based on a comparison of embryo attachment in opossum to 
attachment reaction are homologous processes. That is, these processes evolved from an inflammatory fetal-maternal attachment reaction that likely existed in the therian ancestor.

The difference between the fetal-maternal attachment in marsupials and eutherians is its outcome. In the opossum the brief inflammatory attachment results in parturition, whereas in eutherians it results in implantation and establishment of a sustained fetal-maternal interface.

Here, we elucidate the mechanism by which the ancestral attachment-induced inflammatory response was transformed into the process of embryo implantation in the eutherian lineage. We show that the origin of decidual stromal cells (DSC) - a novel eutherian cell type - was integral to this transformation. First, we provide evidence to further support the homology between opossum attachment reaction and eutherian embryo implantation. Then, we compare gene expression in the uterus of opossum during attachment to that in two eutherians during implantation, armadillo and rabbit. The key differences in gene expression suggest that embryo implantation evolved through suppression of a specific module of the ancestral mucosal inflammatory reaction - neutrophil recruitment mediated by the pro-inflammatory cytokine IL17A. We hypothesized that the origin of DSC in the eutherian lineage (Mess and Carter 2006), coincidentally with embryo implantation, was responsible for the suppression of IL17A in members of this clade. A test of this hypothesis using human cells showed that secretions from DSC inhibit the differentiation of Th17 lymphocytes, the primary producers of IL17A, by inducing a non-standard type-1 interferon response that down-regulates their protein synthesis.

\section{Results and Discussion}

\section{Inflammatory implantation is an ancestral eutherian trait}

The inference of homology between the opossum attachment reaction and eutherian embryo implantation is derived from comparison of opossum to species from Boreotheria (Griffith, Chavan et al. 2017). Boreotheria is one of the three major clades that make up Eutheria and includes primates, rodents, ungulates, carnivores, bats and their kin. However, Eutheria also contains two other major clades, Xenarthra and Afrotheria (dos Reis, Inoue et al. 2012, Tarver, dos Reis et al. 2016), for which data on inflammatory gene expression at implantation was previously unavailable. Xenarthra includes armadillo, sloth, anteater, etc; and Afrotheria includes elephant, hyrax, tenrec, aardvark, dugong, etc. See Figure 1d for the phylogenetic relationship among eutherian species. To test the inference of homology, we investigated the hypothesis that inflammatory implantation is a shared eutherian character; that is, it is not limited to Boreotheria, but is also observed in Xenarthra and Afrotheria. We did this by assessing the expression of marker genes of inflammation during embryo implantation in the nine-banded armadillo (Dasypus novemcinctus) and rock hyrax (Procavia capensis), as representatives of Xenarthra and Afrotheria, respectively. 
bioRxiv preprint doi: https://doi org/10.1101/429571; this version posted September 27, 2018. The copyright holder for this preprint (which was not certified by peer review) is the author/funder, who has granted bioRxiv a license to display the preprint in perpetuity. It is made available under aCC-BY-NC-ND 4.0 International license.

(a) Inflammation mediators during implantation
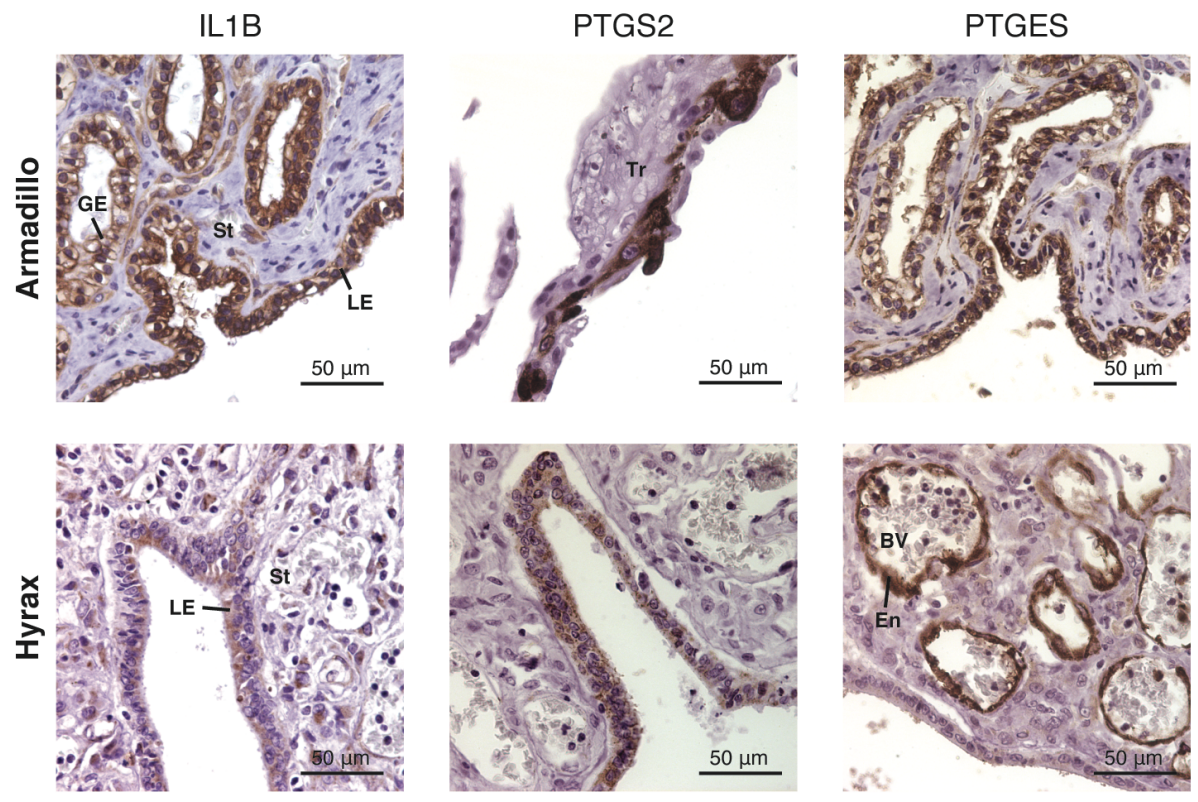

(b) Armadillo: cytokines (mRNA)

(c) Armadillo: genes upregulated at implantation

\begin{tabular}{|c|c|c|}
\hline & $\begin{array}{l}\text { Non } \\
\text { pregnant }\end{array}$ & $\begin{array}{l}\text { Peri } \\
\text { implantation }\end{array}$ \\
\hline IL1A & 1.7 & 39.3 \\
\hline IL1B & 0 & 2.2 \\
\hline TNF & 0 & 0.5 \\
\hline IL1R1 & 6.1 & 31.4 \\
\hline IL6 & 2.3 & 6.4 \\
\hline LIF & 0 & 115.4 \\
\hline PTGS2 & 2.4 & 14.6 \\
\hline PTGES & 1.2 & 100.2 \\
\hline PTGER2 & 6.4 & 18.8 \\
\hline PTGER4 & 18.4 & 18.4 \\
\hline IL8 & 0.6 & 47.2 \\
\hline IL10 & 0 & 15.4 \\
\hline
\end{tabular}

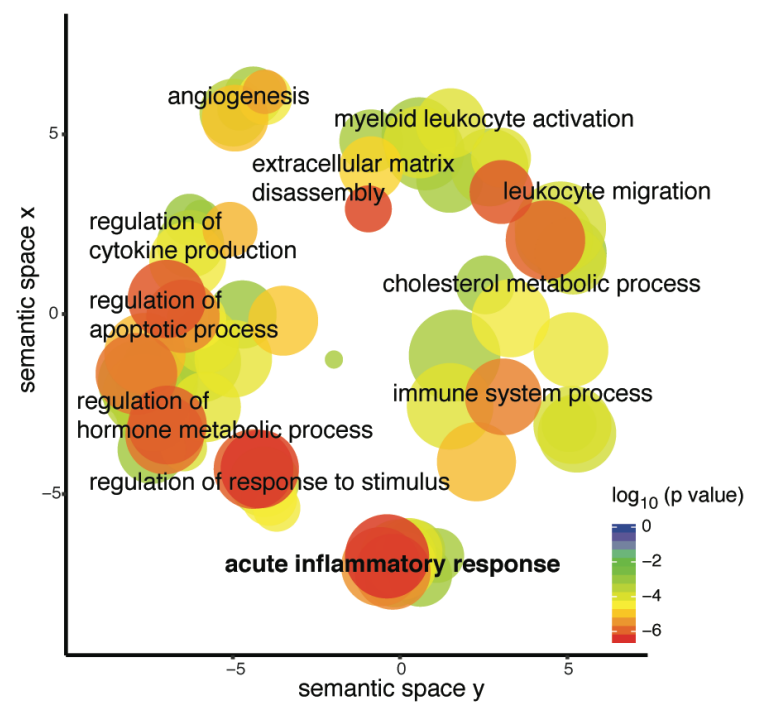

(d) Phylogenetic distribution of inflammation during implantation

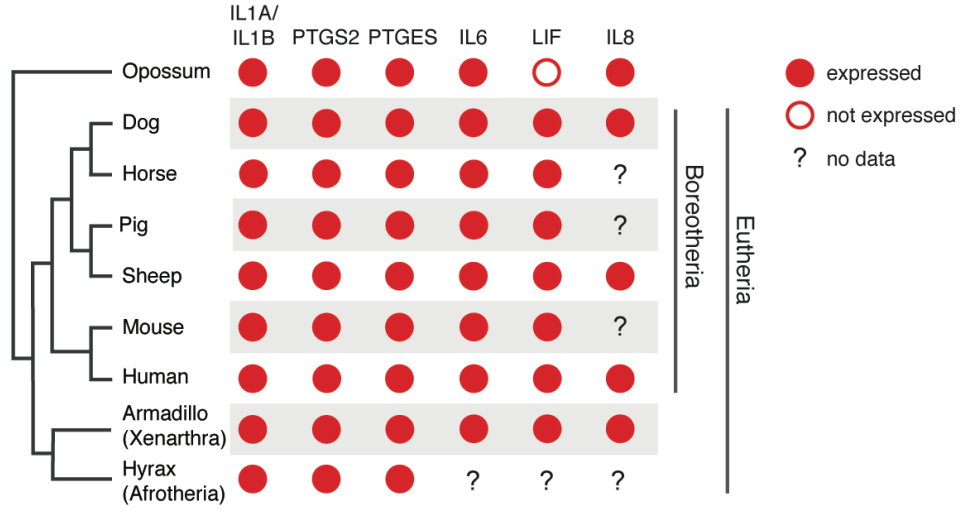


100

101

102

103

104

105

106

107

108

109

110

111

112

113

114

115

116

117

118

119

120

121

122

123

124

125

126

127

128

129

130

131

132

133

134

135

136

137

138

139

Figure 1 Inflammatory implantation is an ancestral eutherian character. (a) Immunohistochemistry for inflammation marker genes IL1B, PTGS2, and PTGES at the fetal-maternal interface in armadillo and hyrax. Nuclei are blue due to hematoxylin counterstaining and the immunostaining signal is brown due to $\operatorname{DAB}($ 3,3'-diaminobenzidine). $\mathrm{GE}=$ glandular epithelium, $\mathrm{LE}=$ luminal epithelium, $\mathrm{St}=$ stroma, $\mathrm{Tr}=$ trophoblast, $\mathrm{BV}=$ blood vessel, En = endothelium. (b) Abundance of mRNA transcripts $(\mathrm{TPM}=$ Transcripts per Million) of key inflammatory genes in armadillo uterus in non-pregnant and periimplantation stage. (c) Enriched gene ontology (GO) categories among the genes that are upregulated at least 10 -fold in armadillo uterus in transition from non-pregnant to peri-implantation stage. GO categories are clustered by semantic similarity. GO categories closer to each other are semantically similar; those represented by red circles are more significantly enriched than those in blue. (d) Comparison of expression of inflammatory genes during embryo attachment or implantation in therian mammals. Data for human: cytokines (reviewed in Van Sinderen, Menkhorst et al. 2013), PTGS2 (Marions and Danielsson 1999), PTGES (Milne, Perchick et al. 2001). Data for sheep, horse, pig, dog, and mouse: (reviewed in Chavan, Griffith et al. 2017).

One of the earliest signals of inflammation, IL1B, is expressed during implantation in the luminal epithelium of the endometrium in armadillo and hyrax (Figure 1a). PTGS2 and PTGES, enzymes involved in the synthesis of prostaglandin $\mathrm{E}_{2}\left(\mathrm{PGE}_{2}\right)$, are also expressed during implantation in both species, although the tissues in which these genes are expressed differ between species. PTGS2 and PTGES are expressed on the two sides of the fetal-maternal interface in armadillo - PTGS2 in the trophoblast, while PTGES in the endometrial luminal epithelium. In hyrax, they are both expressed on the maternal side, with PTGS2 in the luminal epithelium and PTGES in the endothelia within the endometrium.

Next, we used transcriptome data to test whether there is a broad signature of inflammation during implantation in armadillo uterus. A variety of inflammatory genes are up-regulated during armadillo implantation: cytokines such as IL1A, IL1B, IL6, LIF, IL8 (CXCL8), and IL10; cytokine receptor ILIR1; prostaglandin synthesis enzymes PTGS2 and PTGES; and prostaglandin receptors PTGER2 and PTGER4 (Figure 1b). None of the cytokines shown in Figure $\mathbf{1 b}$ is expressed in the non-gravid uterus, i.e. their mRNA abundance is below the operational threshold of 3 TPM (Wagner, Kin et al. 2013), but most of them are expressed highly during implantation. Genes up-regulated more than 10-fold in the transition from non-pregnant to implantation stage are significantly enriched in Gene Ontology (GO) categories related to inflammation (Figure 1c), such as acute inflammatory process, immune system process, leukocyte migration, regulation of cytokine production, and regulation of response to stimulus.

We summarized the above expression pattern of inflammation marker genes during implantation on a phylogeny of therian mammals, along with the expression patterns from other representative eutherian species, and from a marsupial, opossum, at the time of attachment between fetal and maternal tissues at 13.5 days post-copulation (dpc) (Figure 1d). In all major clades of Eutheria, the uterine changes during embryo implantation closely resemble an acute inflammatory reaction. Parsimoniously, this suggests that embryo attachment-induced uterine inflammatory signaling is a plesiomorphic trait of eutherian mammals, i.e. a trait that was inherited from an 
140 ancestral lineage and shared with the sister group, the marsupials, adding further support to the

141 argument that attachment-induced inflammation of marsupials is homologous to eutherian

142 embryo implantation (Griffith, Chavan et al. 2017, Griffith, Chavan et al. 2018, Liu 2018). In

143 other words, eutherian implantation likely evolved from, and through modification of, ancestral

144 attachment-induced inflammation.

145 Differences in uterine gene expression between opossum and eutherians

146 To understand how eutherian implantation evolved from the ancestral therian attachment induced 147 inflammation, we compared uterine gene expression during attachment-induced inflammation in 148 opossum (Monodelphis domestica) to that during implantation in two eutherian mammals, 149 armadillo (Dasypus novemcinctus) and rabbit (Oryctolagus cuniculus) (rabbit data from Liu, 150 Zhao et al. 2016). Gene expression patterns shared by armadillo and rabbit are likely to be shared 151 by eutherians in general since armadillo and rabbit are phylogenetically positioned so that their 152 common ancestor is the common ancestor of all extant eutherian mammals.

153 In the uterine transcriptomes of opossum, armadillo, and rabbit, we classified each gene as either 154 expressed or not expressed (see Methods). We then classified these genes as "opossum-specific" 155 if they are expressed in opossum but not expressed in armadillo and rabbit, and "Eutheria156 specific" if they are not expressed in opossum but are expressed in both armadillo and rabbit. 157 There are 446 and 456 genes in these categories, respectively, among the total of 11,089 genes 158 that have one-to-one orthologs in all three species.

159 First we identified enriched GO categories in the opossum-specific and Eutheria-specific lists of 160 genes (Figure 2). To increase the specificity of GO enrichment analysis, we used only the subset 161 of the opossum-specific expressed genes that have an at least 2-fold higher gene expression in 162 the attachment phase compared to the non-pregnant stage (204 genes). Such refinement of the 163 Eutheria-specific gene set was not possible since we do not have a transcriptome of non-pregnant 164 rabbit uterus. The opossum-specific set is enriched for genes related to lipid metabolism, 165 especially mobilization of fatty acids from cell membrane, and lipid transport. Lipid metabolism 166 genes are also upregulated in the pregnant uterus of another marsupial, fat-tailed dunnart 167 (Sminthopsis crassicaudata) (Whittington, O'Meally et al. 2018). These genes may have 168 functions related to nutrient transfer or steroid metabolism but lipid metabolism is also important 169 in inflammation: for example, the first step in the synthesis of prostaglandins is to break down 170 membrane triglycerides. The opossum-specific set is also enriched for GO category "cellular 171 response to Interleukin-1", i.e. genes downstream of IL1A and IL1B. This suggests that although 172 inflammatory signaling is initiated by IL1A and/or IL1B upon embryo attachment in opossum as 173 well as in eutherians, their downstream targets are activated only in the opossum. This 174 observation recapitulates at the molecular level a phenomenon at the organismal level 175 inflammatory signaling is observed in both, but has different outcomes of parturition and 176 implantation in opossum and eutherians respectively. 
(a) Opossum-specific genes

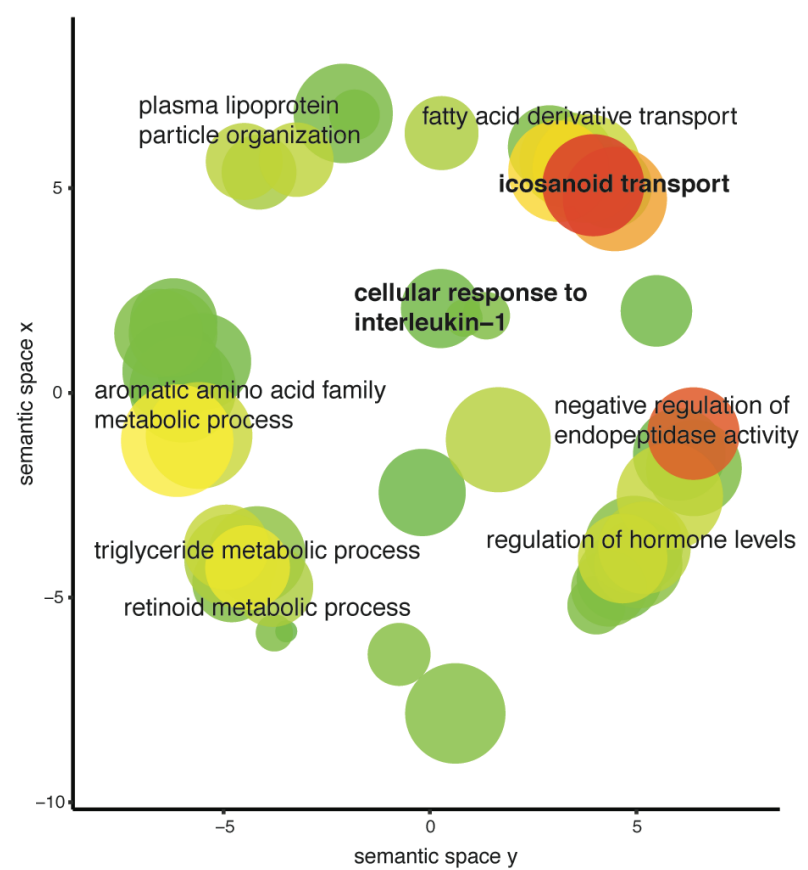

(b) Eutheria-specific genes

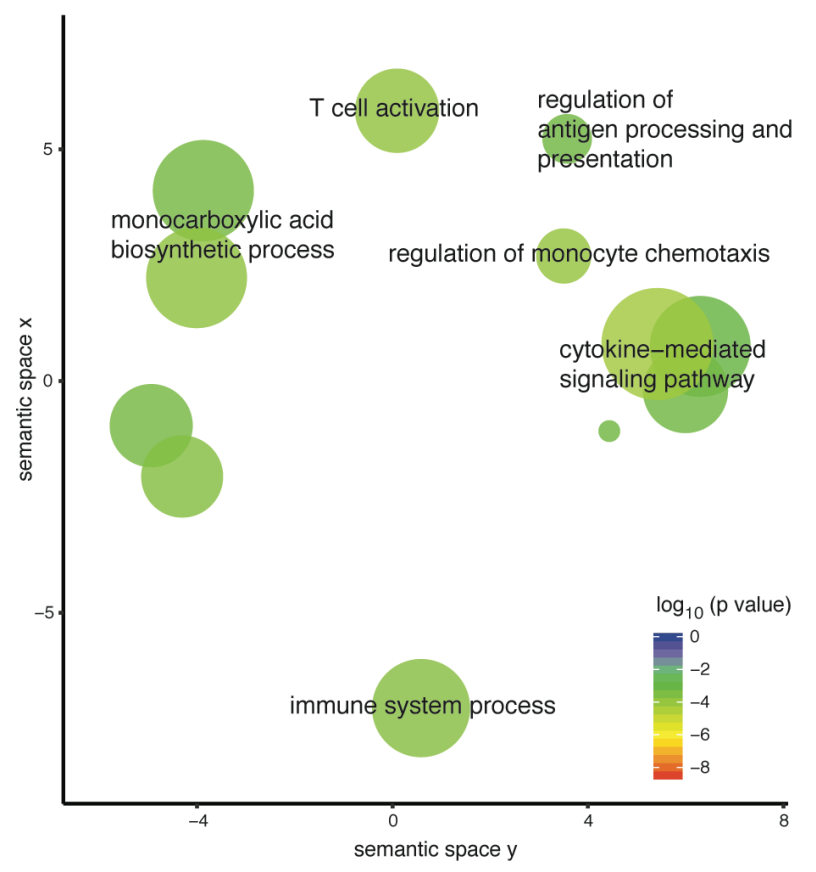

Figure 2 Transcriptomic differences between marsupial and eutherian attachment reaction. Genes were classified as opossum-specific (a) and eutherian-specific (b). GO categories enriched in each set are shown in the figures, where semantically similar categories are clustered together in space.

\section{IL17A expression was suppressed in Eutheria}

IL1A and IL1B set off a cascade of inflammatory signaling events mediated by cytokine molecules. Therefore, in order to identify the specific differences between opossum and eutherians in response to interleukin-1, we looked for differences in the expression pattern of cytokines (Figure 3a). Cytokines were identified as genes assigned to GO category "cytokine activity" (GO:0005125).

Eutheria-specific expressed cytokines are CXCL10, CCL5, NDP, WNT7A, and LIF. The first two, CXCL10 and CCL5 attract leukocytes such as T cells, NK cells, and dendritic cells to the sites of inflammation (Schall 1991, Dufour, Dziejman et al. 2002). NDP and WNT7A are both members of the Wnt signaling pathway, which is important for communication between implanting blastocyst and endometrium (Wang and Dey 2006, Chen, Zhang et al. 2009, Sonderegger, Pollheimer et al. 2010); inhibition of this process prevents successful implantation in mouse (Mohamed, Jonnaert et al. 2005). LIF is a critical signaling molecule in eutherian mammals for the differentiation of decidual stromal cells from endometrial stromal fibroblasts; and its expression is therefore obligatory for successful implantation (Shuya, Menkhorst et al. 2011). This set of genes represents cytokines and signaling molecules that were likely recruited within the eutherian lineage to enable decidual cell differentiation, embryo-uterine crosstalk, regulation of leukocyte traffic, and therefore implantation. 
(a) Cytokines

\begin{tabular}{|c|c|c|c|c|}
\hline IL1B & 3.6 & 4.2 & 5.8 & \multirow{2}{*}{ tpm 900} \\
\hline IL8 & 16 & 90.1 & 6.2 & \\
\hline LTB & 11.9 & 10.7 & 18.4 & \multirow{4}{*}{400} \\
\hline IL15 & 6.8 & 61.1 & 19.9 & \\
\hline EDN1 & 21.4 & 6.3 & 24.1 & \\
\hline WNT5A & 23.7 & 9.7 & 34.2 & \\
\hline CX3CL1 & 10.2 & 32.3 & 42.9 & \multirow{2}{*}{100} \\
\hline BMP4 & 28.1 & 155.2 & 70.8 & \\
\hline TNFSF13 & 9.3 & 82.5 & 99.8 & \multirow{4}{*}{0} \\
\hline CSF1 & 7 & 42.2 & 100.2 & \\
\hline TNFSF10 & 51.7 & 13.2 & 333.1 & \\
\hline CXCL12 & 49.1 & 27.6 & 862.8 & \\
\hline CXCL10 & 0.9 & 12 & 17.3 & \multirow{23}{*}{$\begin{array}{l}\text { Eutheria } \\
\text { specific }\end{array}$} \\
\hline CCL5 & 1.2 & 54.5 & 5.9 & \\
\hline NDP & 0.4 & 55.3 & 26.4 & \\
\hline LIF & 1.5 & 220.1 & 10.7 & \\
\hline WNT7A & 0.2 & 787.9 & 93.2 & \\
\hline CTF1 & 37.1 & 1.1 & 3.1 & \\
\hline KITLG & 19.2 & 2.5 & 3.9 & \\
\hline TNFSF15 & 26.4 & 0 & 4.7 & \\
\hline CCL19 & 6.5 & 0 & 6.1 & \\
\hline IL16 & 3 & 2.9 & 22.4 & \\
\hline IL17D & 5.9 & 2.4 & 36.2 & \\
\hline CCL21 & 92.6 & 0.1 & 215.2 & \\
\hline INHBA & 5.2 & 0.1 & 348.2 & \\
\hline CCL17 & 0.1 & 0 & 6.2 & \\
\hline CCL25 & 0.6 & 0 & 12.7 & \\
\hline WNT2 & 0.3 & 3 & 40.2 & \\
\hline FGF2 & 1.5 & 1.7 & 92.4 & \\
\hline IL17B & 2.9 & 0.5 & 99.4 & \\
\hline IL20 & 0.9 & 0.5 & 963.2 & \\
\hline IL1A & 48.2 & 74.9 & 0.3 & \\
\hline IL10 & 157.9 & 29.3 & 2.9 & \\
\hline LTA & 0.1 & 3.1 & 0 & \\
\hline CXCL9 & 0.9 & 5 & 1.5 & \\
\hline CSF3 & 3.6 & 0 & 0.3 & \multirow{5}{*}{$\begin{array}{l}\text { Opossum } \\
\text { specific }\end{array}$} \\
\hline CCL20 & 11.5 & 0 & 0 & \\
\hline & 51.6 & 1 & 1.7 & \\
\hline \multirow[t]{2}{*}{ IL17A } & 79.1 & 0 & 0 & \\
\hline & Opossum & Armadillo & Rabbit & \\
\hline
\end{tabular}

(b) IL17A in opossum uterus

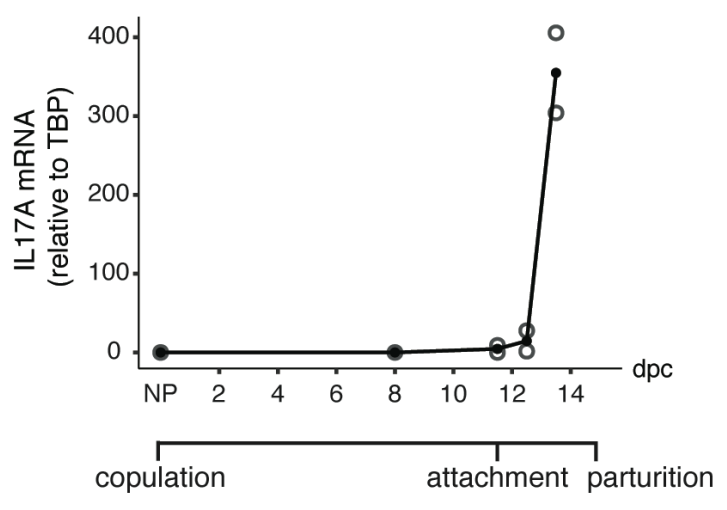

(c) Neutrophils in opossum uterus at $14 \mathrm{dpc}$

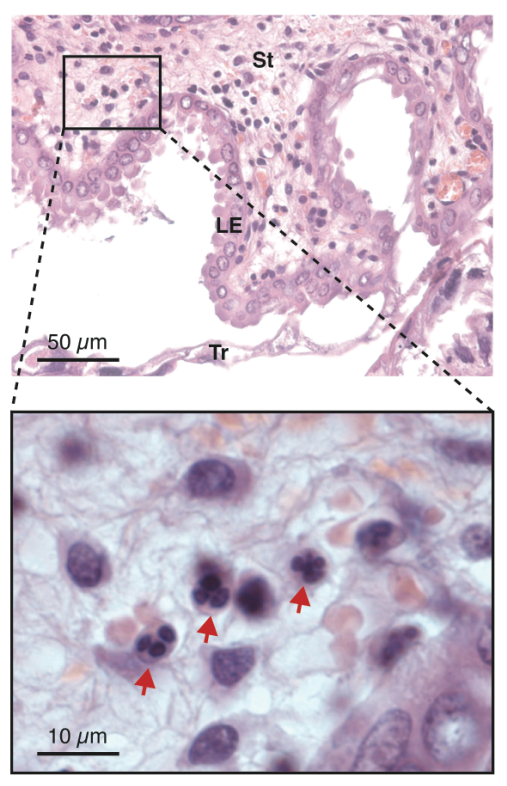

Figure 3 IL17A signaling is suppressed in eutherians. (a) Gene expression of cytokines during embryo attachment or implantation in opossum, armadillo, and rabbit uterus. Intensity of red is proportional to the abundance of mRNA. Genes expressed below 3 TPM are considered unexpressed (Wagner, Kin et al. 2013). Rows are ordered by the gene expression patterns across species: expressed in all species, expressed only in Eutheria, expressed in opossum and rabbit, rabbit-specific, expressed in opossum and armadillo, armadillo-specific, and opossum-specific. Cytokines not expressed in any of these species are not shown in the figure. (b) Expression of $I L 17 A$ through the pregnancy of opossum, measured relative to $T B P$, by qPCR. Embryo attachment begins around 11.5 days post-copulation (dpc), and pregnancy ends at $14.5 \mathrm{dpc}$. (number of biological replicates: $\mathrm{NP}=3,8 \mathrm{dpc}=3,11.5 \mathrm{dpc}=2,12.5 \mathrm{dpc}=2,13.5 \mathrm{dpc}=2$ ) (c) Neutrophils infiltration in H\&E stained opossum uterus at $14 \mathrm{dpc}$, indicated by red arrows in the zoomed in micrograph. $\mathrm{LE}=$ luminal epithelium, $\mathrm{St}=$ stroma, $\mathrm{Tr}=$ trophoblast. $\mathrm{TPM}=$ Transcripts per Million. 
212 The inverse set, opossum-specific cytokines, includes CSF3, CCL20, TNF, and IL17A. CSF3,

213 also known as G-CSF (Granulocyte Colony Stimulating Factor) attracts granulocytes (Lieschke,

214 Grail et al. 1994, Panopoulos and Watowich 2008), such as neutrophils, and is positively

215 regulated by IL17A (Ye, Rodriguez et al. 2001, Onishi and Gaffen 2010). CCL20 attracts

216 lymphocytes as well as neutrophils and also is a downstream gene of IL17A (Onishi and Gaffen

217 2010). This pattern is indicative of a mucosal inflammatory reaction, culminating with

218 recruitment of effector cells such as neutrophils.

219 Since IL17A is upstream of the other genes in the opossum-specific set, we then measured the

220 expression of IL17A in opossum uterus by qPCR to test whether it is induced in response to the

221 attaching embryo or expressed throughout pregnancy. Figure 3b shows that IL17A is not

222 expressed in non-pregnant and $8 \mathrm{dpc}$ uteri, and only begins expression at $11.5 \mathrm{dpc}$, coincidental

223 with the egg-shell breakdown and beginning of fetal-maternal attachment. Its expression reaches

224 a very high level by $13.5 \mathrm{dpc} ; 79$ TPM according to the transcriptomic data. This time-course

225 expression data suggests that $I L 17 A$ is induced specifically in response to embryo attachment in

226 opossum, even though it is completely absent in armadillo and rabbit during implantation (0

227 TPM in both species). One of the hallmarks of IL17A-mediated inflammation - through the

228 action of cytokines like CXCL8, CSF3, CCL20 - is neutrophil infiltration into the inflamed

229 tissue (Medzhitov 2007, Onishi and Gaffen 2010, Griffin, Newton et al. 2012, Pappu, Rutz et al.

230 2012, Flannigan, Ngo et al. 2016). Therefore, we tested whether IL17A expression in opossum is

231 also accompanied by neutrophil infiltration. Neutrophils are not seen in early stages of

232 pregnancy, but at $14 \mathrm{dpc}$, neutrophil infiltration is clearly seen histologically (Figure 3c).

233 Consistent with the pattern of $I L 17 \mathrm{~A}$ expression, neutrophils are absent from the fetal-maternal

234 interface at the time of implantation in eutherian mammals (evidence reviewed in Chavan,

235 Griffith et al. 2017).

236 The absence of $I L 17 A$ expression in eutherian mammals at implantation is likely to be due to its

237 loss in the eutherian lineage rather than its recruitment in the marsupial lineage. The discovery of

238 IL17 homologs as the early-responding cytokines in the sea urchin larva during gut infection

239 (Buckley, Ho et al. 2017) suggests that it may be an ancient mucosal inflammatory cytokine at

240 least as old as deuterostomes. Since the endometrium is a mucosal tissue, IL17A - a key

241 mucosal inflammatory cytokine - is likely to have been expressed in the ancestral therian

242 endometrium during attachment-induced inflammation, and its expression was lost later during

243 evolution in the eutherian lineage.

244 Because $I L 17 A$ is 1) the most highly expressed gene among opossum-specific cytokines, 2) an 245 important regulator of mucosal inflammation, 3) upstream of chemokines like CSF3, and 4) not

246 expressed in armadillo and rabbit at all, even in a leaky manner, we posited that the loss of IL17A

247 expression - and thus the loss of neutrophil infiltration - after embryo attachment was one of 
the key innovations that transformed the ancestral inflammatory attachment reaction into embryo implantation.

Contrary to the data from armadillo and rabbit, some IL17A expression has been reported at the fetal-maternal interface in mouse and human, specifically in the $\gamma \delta \mathrm{T}$ cells in the mouse (Pinget, Corpuz et al. 2016). However, functional evidence suggests that even in mouse and human, an induction of IL17A expression at the fetal-maternal interface is detrimental to pregnancy. The strongest evidence perhaps comes from mouse, where injection of polyI:C to mimic viral infection during pregnancy induces the expression of IL17A in the decidua. Maternal IL17A then makes its way into the fetal circulation, interferes with brain development of the embryos, and leads to behavioral abnormalities resembling Autism Spectrum Disorder (ASD) in pups (Choi, Yim et al. 2016). Li and colleagues ( $\mathrm{Li}, \mathrm{Qu}$ et al. 2018) showed that in a mouse model of spontaneous abortion, Th17 cell numbers are higher in the decidua relative to normal pregnancy. In human, Th17 levels in peripheral blood are elevated in women with recurrent spontaneous abortions compared to women with healthy pregnancy (Wang, Hao et al. 2010). Nakashima and colleagues (Nakashima, Ito et al. 2010) found that while IL17A-positive cells were only occasionally present in the deciduae of normal pregnancies, their numbers were significantly elevated in pregnancies with first trimester spontaneous abortions. These studies clearly indicate that IL17A signaling at the fetal-maternal interface is not conducive to successful pregnancy, and the cases in human and mouse where IL17A expression is observed, the downstream signaling is likely inhibited in some way.

Next, we sought to identify the mechanism by which IL17A signaling was suppressed in the evolution of eutherian mammals.

\section{Decidual stromal cells suppressed IL17A expression at implantation in Eutheria}

Decidual stromal cells (DSC) are a novel cell type that originated in the stem lineage of eutherian mammals (Mess and Carter 2006, Wagner, Kin et al. 2014, Erkenbrack, Maziarz et al. 2018). They differentiate from endometrial stromal fibroblasts (ESF) in many eutherian mammals during pregnancy (Gellersen and Brosens 2014) and also during the menstrual cycle in primates, some bats, the elephant shrew (Emera, Romero et al. 2011), and the spiny mouse (Bellofiore, Ellery et al. 2017) in a process called decidualization. The evolution of DSC from ancestral therian ESF was associated with modulation of expression of genes involved in the innate immune response (Kin, Maziarz et al. 2016). DSC perform many functions critical to the maintenance of pregnancy in human and mouse, for example, regulation of the traffic of leukocytes into the endometrium during pregnancy (Nancy, Tagliani et al. 2012, Erlebacher 2013), production of hormones like prolactin, regulation of invasiveness of the trophoblast (Gellersen and Brosens 2014), regulating communication between cell types at the fetal-maternal interface (Pavlicev, Wagner et al. 2017). Outside of euarchontoglirean mammals (primates, rodents and their relatives), however, DSC are not maintained throughout the pregnancy. In bats 
285 (Laurasiatheria), hyrax, tenrec (Afrotheria), and armadillo (Xenarthra, also see Supp. Figure 1), 286 DSC differentiate around the time of implantation but are often lost soon after implantation. This

287 suggests that the ancestral function of DSC when they originated, was likely to have been limited 288 to the time of implantation (Chavan, Bhullar et al. 2016). Based on their inferred ancestral role at 289 the time of implantation (Chavan, Bhullar et al. 2016), their ability to regulate the immune 290 response during pregnancy (Erlebacher 2013), and because their origin in the eutherian stem 291 lineage (Mess and Carter 2006) coincides with the evolution of suppression of IL17A expression 292 (this study), we hypothesized that DSC played a role in the regulation of IL17A during 293 implantation.

294 IL17A is typically expressed by Th17 cells, which differentiate from naïve T cells in the 295 presence of IL6 and TGFB1 (Bettelli, Carrier et al. 2006) (Figure 4a). We hypothesized that 296 DSC suppress IL17A expression by inhibiting the differentiation of naïve T cells into Th17 cells. 297 To test this hypothesis, we differentiated primary human naïve T cells into Th17, in the presence 298 of DSC-conditioned or control medium and measured IL17A secreted by T cells with ELISA. 299 Treatment of differentiating $\mathrm{T}$ cells with DSC conditioned medium decreases their IL17A 300 production significantly ( 2 fold, $\mathrm{p}<10^{-6}$ ), while treatment with unconditioned DSC medium has 301 no effect. The decreased level of IL17A is not statistically distinguishable from that in 302 unstimulated naïve $\mathrm{T}$ cells, suggesting that DSC-conditioned medium completely suppresses 303 upregulation of IL17A production during Th17 differentiation (Figure 4b; see Supp. Figure 2 304 for mRNA levels of $I L 17 A)$.

$305 \mathrm{Wu}$ and colleagues (Wu, Jin et al. 2014) showed that Th17 cells are present in the human 306 endometrium during the first trimester and that they are recruited there by DSC, which appears to 307 contradict what we have shown in this study. However, the Th17 cells reported in the decidua by $308 \mathrm{Wu}$ and colleagues are CD45RO+, i.e. they are memory Th17 cells that are already 309 differentiated, while we show that DSC inhibit the differentiation of Th17 cells from naïve T 310 cells. 
(a) Regulation of Type 17 response

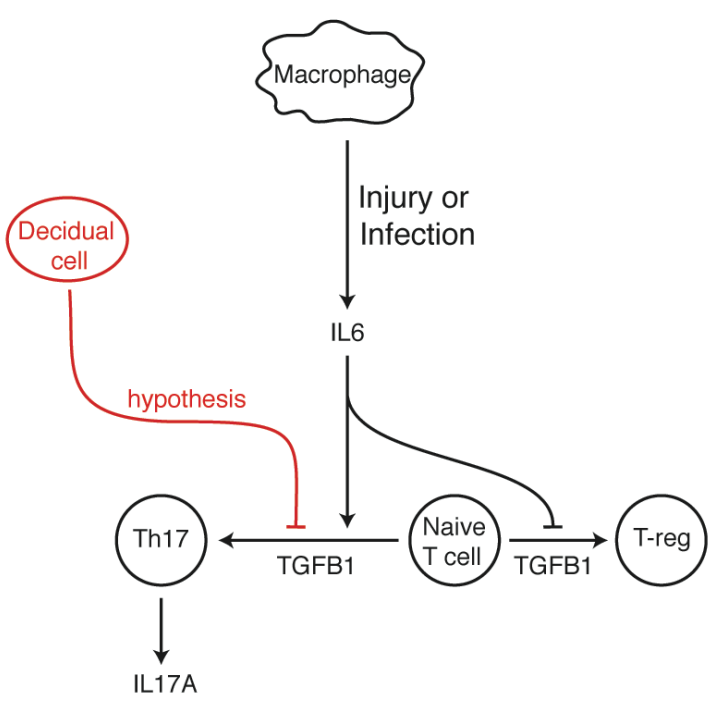

(b) Test of hypothesis

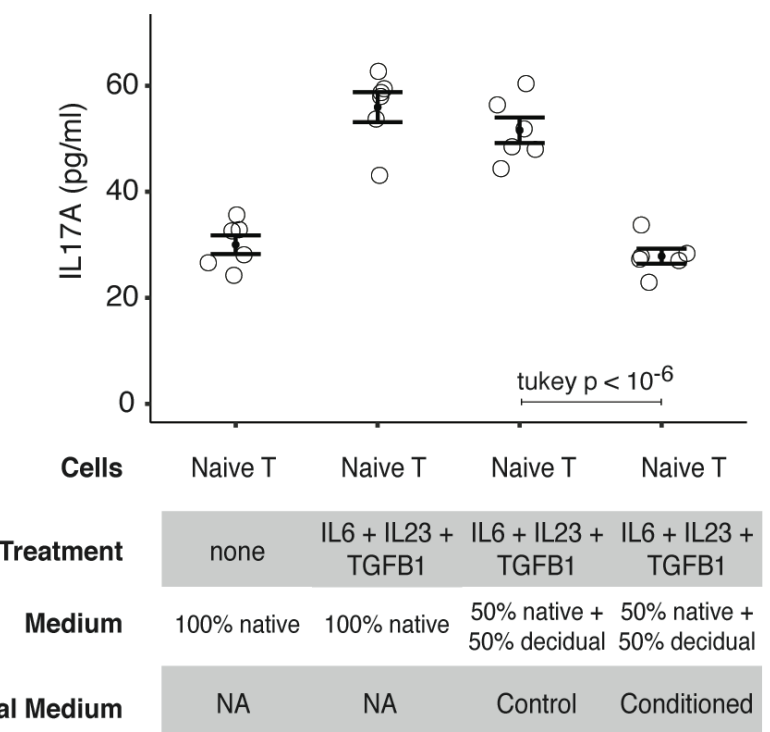

311

Figure 4 Decidual stromal cells suppress Th17 cell differentiation. (a) Schematic showing how Th17 cells differentiate from naïve T cells, and the hypothesis for the role of DSC (b) Test of the hypothesis using in-vitro differentiation of human naïve T cells into Th17 cells. IL17A secretion ( $\mathrm{pg} / \mathrm{ml}$ ) by Th17 cells is shown (mean and standard error of the mean). The first two samples are reference points, where Th17 cells were differentiated in their native medium, and the last two samples were differentiated in DSC control or conditioned medium.

\section{DSC inhibit protein synthesis during Th17 differentiation}

To understand how DSC suppress differentiation of naïve T cells into Th17 cells, we sequenced the transcriptomes of the differentiating $\mathrm{T}$ cells that were either treated with DSC conditioned medium or control medium (conditions 3 and 4 from Figure 4b). Principal components analysis of the transcriptome data shows that the DSC conditioned medium had a systematic effect on the gene expression profile of the $\mathrm{T}$ cells since $60 \%$ of the variance between samples can be explained by the first principal component along which the samples separate by treatment (Figure 5a). 
(a) Principal Components Analysis

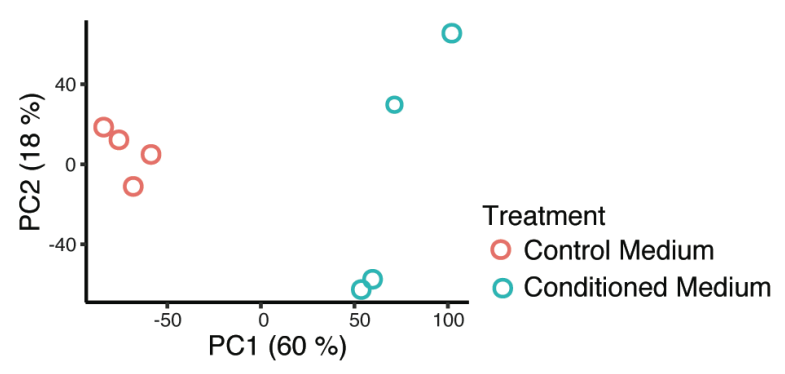

(b) $\mathrm{CPM}_{\text {conditioned }}>\mathrm{CPM}_{\text {control }}$

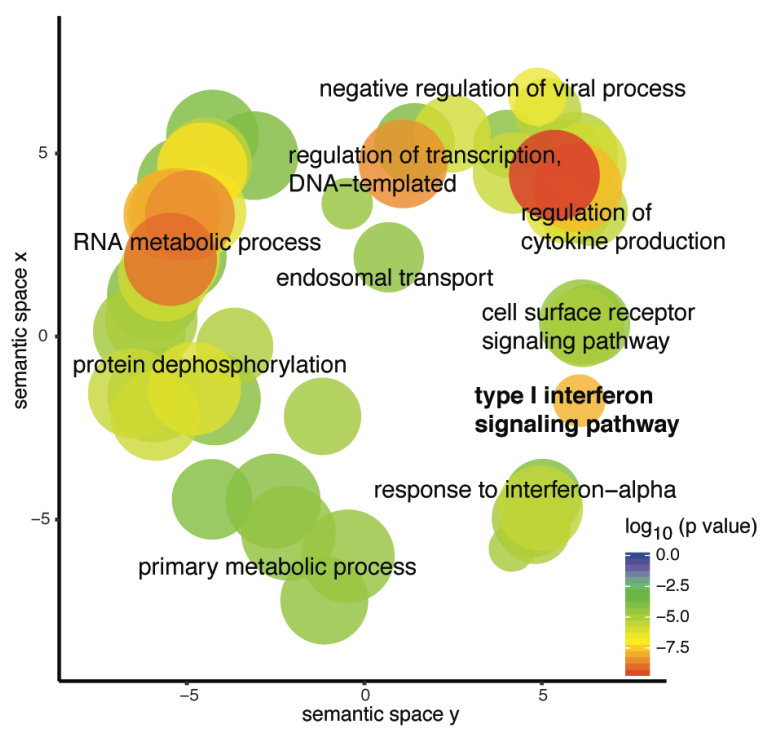

(c) $\mathrm{CPM}_{\text {control }}>\mathrm{CPM}_{\text {conditioned }}$

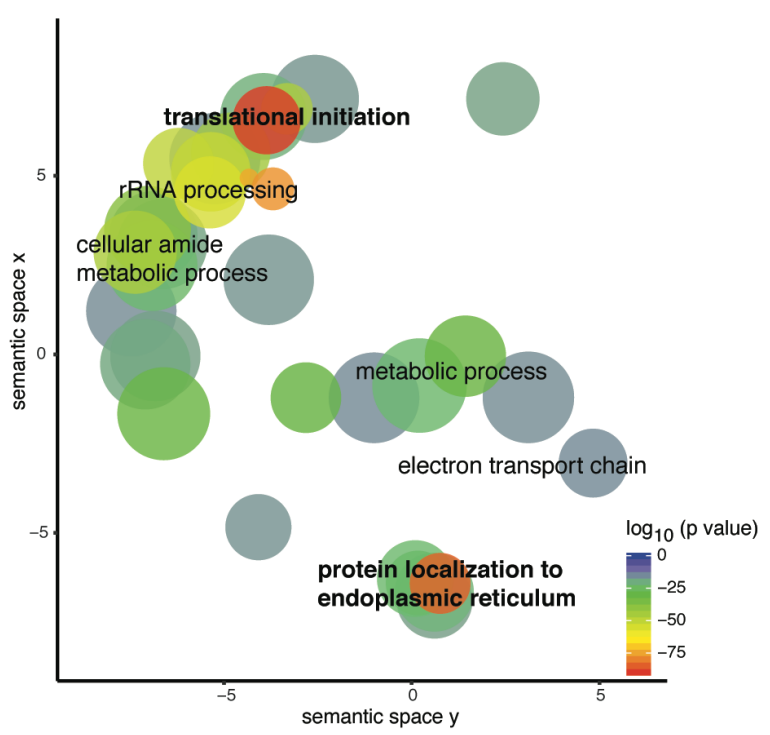

Figure 5 RNA-seq of Th17 cells differentiated in control vs. DSC conditioned medium. (a) Principal components analysis of transcriptomes of the control and conditioned medium treated cells. Square root of CPM was used for this analysis. (b) and (c) respectively show GO categories enriched in genes that have higher expression in T cells treated with conditioned medium compared to the control medium, and vice versa. $\mathrm{CPM}=$ Counts per Million.

Genes significantly up-regulated, as assessed by EdgeR (Robinson, McCarthy et al. 2010), in T cells treated with DSC conditioned medium compared to $\mathrm{T}$ cells treated with control medium are enriched for GO categories related to defense of viral infection, such as the type-1 interferon signaling pathway, negative regulation of viral process, response to interferon alpha, RNA metabolic process (Figure 5b). The down-regulated genes are enriched for GO categories related to protein synthesis and secretion, e.g. rRNA processing, translation initiation, protein localization to endoplasmic reticulum. Genes of the electron transport chain are also downregulated, suggesting that the cells down-regulated their ATP synthesis and became quiescent (Figure 5c).

Then we looked more specifically at genes in the type-1 interferon signaling pathway (Figure 6a). Cells infected with virus produce type- 1 interferons (IFN- $\alpha$ and IFN- $\beta$ ), which regulate gene expression in an autocrine and paracrine manner via binding to type-1 interferon receptors 
344 IFNAR1 and IFNAR2. Curiously, none of the IFN- $\alpha$ genes is expressed in either samples, and 345 IFN- $\beta$ gene $(I F N B)$ is barely expressed in both samples $(<1$ TPM) but is not differentially 346 expressed in response to DSC conditioned medium. Both IFN receptor genes, however, are 347 significantly up-regulated in samples treated with conditioned medium (Figure 6b). Importantly, 348 none of the type-1 interferons are expressed in DSC either (Figure 6b). This suggests that the 349 activation of type-1 interferon signaling in $\mathrm{T}$ cells is in response to a specific non-interferon 350 signal from DSC and not a genuine viral defense response.

351 One of the prominent outcomes of type-1 interferon signaling is inhibition of protein synthesis 352 via mRNA degradation and inhibition of translation initiation machinery (Kindt, Goldsby et al. 353 2007). This ensures that viral nucleic acids do not replicate within the host cells and produce 354 more viral particles. In T cells treated with DSC-conditioned medium, three out of four 2'-5' 355 oligoadenylate synthase genes $(O A S 1, O A S 2$ and $O A S 3)$ genes are up-regulated (Figure 6c). 356 These genes activate RNaseL, which in turn degrades mRNA. Most of the eukaryotic translation 357 initiation factors (EIFs) are also down-regulated and at the same time two negative regulators of 358 EIFs, viz. EIF2AK2 and EIF4EBP2 are up-regulated (Figure 6d), indicating decreased protein 359 synthesis.

360 Together these observations suggest that DSC suppress Th17 differentiation by inducing type-1 361 interferon signaling, which consequently decreases overall protein synthesis and ATP synthesis. 362 However, DSC secrete neither type-1 IFNs nor do they induce the expression of type-1 IFNs in T 363 cells, suggesting that DSC hijack the viral defense mechanism downstream of the IFN receptors 364 to inhibit protein synthesis in T cells. This is in contrast to the bovine pregnancy in which the ruminant-specific type-1 interferon IFN- $\tau$ (IFNT) from the embryo suppresses IL17A expression 366 in maternal peripheral blood mononuclear cells (Talukder, Rashid et al. 2018). Signals from DSC 367 that inhibit protein synthesis in human $\mathrm{T}$ cells are unlikely to be generic molecules that can 368 induce type-1 IFN signaling, e.g. cell free RNA or DNA. First of all, such molecules would 369 induce type-1 IFN signaling in all cells at the fetal-maternal interface, which would be 370 detrimental to the maintenance of pregnancy, just as a viral infection is (Yockey and Iwasaki 371 2018, Yockey, Jurado et al. 2018). A generic signal would also suppress differentiation of naïve $372 \mathrm{~T}$ cells into not only Th17 cells but also other effector T cells. However, T-reg cells are 373 necessary for maintenance of pregnancy (Aluvihare, Kallikourdis et al. 2004), and their 374 differentiation should not be hindered by DSC. Potential signals from DSC that may be acting on 375 T cells are discussed in the supplementary material (Supp. Figure 3). 
(a) Type 1 IFN signaling

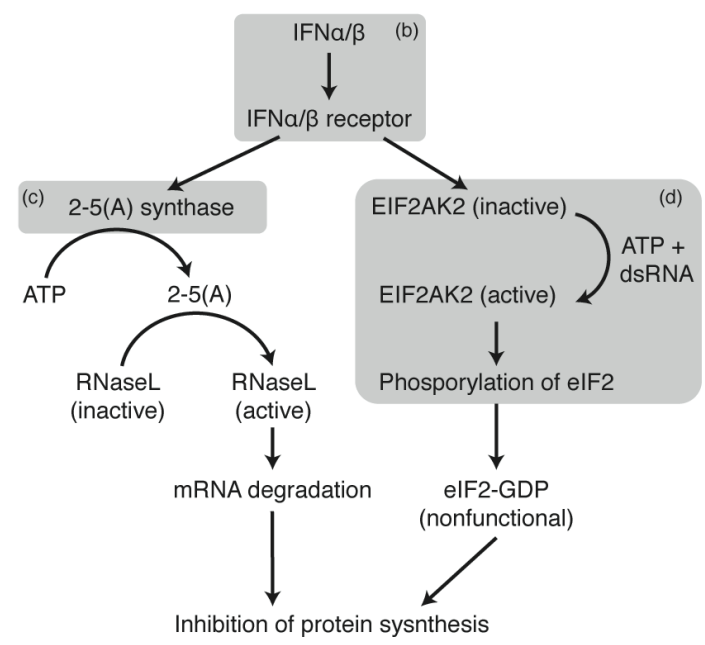

(c) 2'-5' Oligoadenylate synthases

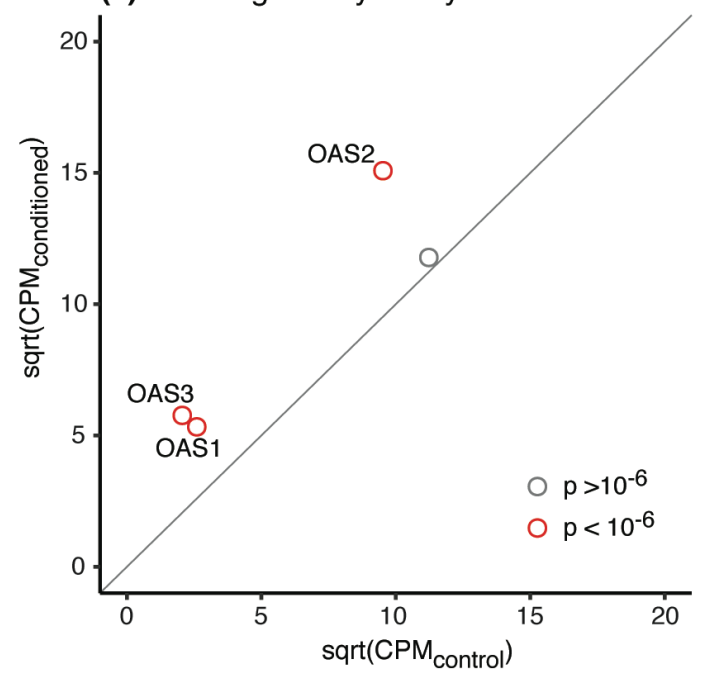

(b) Type 1 IFN genes and receptors

\begin{tabular}{r|c|c|c|c|c|c} 
& \multicolumn{2}{c}{ T cells } & \multicolumn{3}{c}{ Endometrial Stromal Cells } \\
IFNAR2 & 27.2 & 40.5 & $p<10^{-6}$ & 9.1 & 9.7 & \\
IFNAR1 & 36.4 & 60.6 & 104.8 & 76.9 & $p<10^{-6}$ \\
IFNB1 & 0.6 & 0.8 & 0 & 0 & \\
IFNA21 & 0 & 0 & 0 & 0 & \\
IFNA17 & 0 & 0 & 0 & 0 & \\
IFNA16 & 0 & 0 & 0 & 0 & \\
IFNA14 & 0 & 0 & 0 & 0 & \\
IFNA10 & 0 & 0 & 0 & 0 & \\
IFNA8 & 0 & 0 & 0 & 0 & \\
IFNA7 & 0 & 0 & 0 & 0 & \\
IFNA6 & 0 & 0 & 0 & 0 & \\
IFNA5 & 0 & 0 & 0 & 0 & CPM \\
IFNA4 & 0 & 0 & 0 & 0 & -100 \\
IFNA2 & 0 & 0 & 0 & 0 & -75 \\
IFNA1 & 0 & 0 & 0.1 & 0 & -50 \\
& Control Conditioned & ESF & DSC & 0
\end{tabular}

\section{(d) Translation initiation factors}

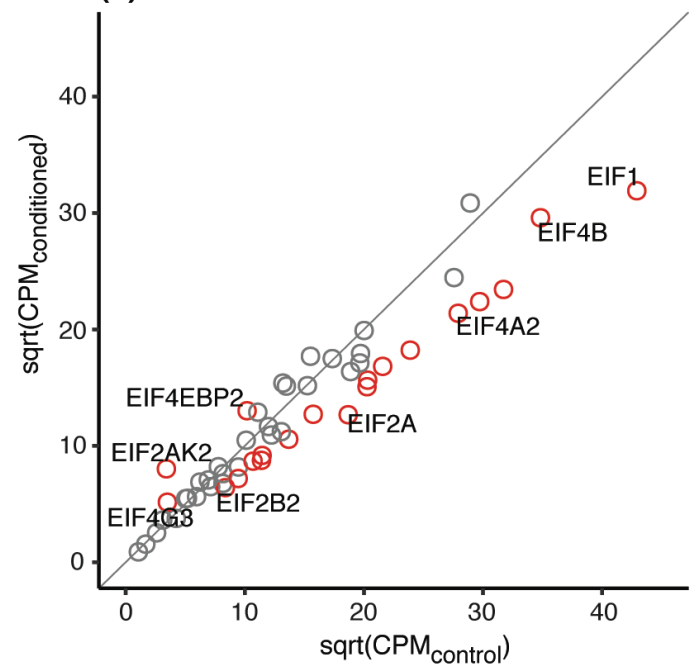

Figure 6 Interferon signaling genes in T cells treated with DSC conditioned medium. (a) Schematic showing how type-1 interferon signaling pathway inhibits protein synthesis, adapted from (Kindt, Goldsby et al. 2007). Grey boxes show the sets of genes from this pathway whose gene expression is shown in the following panels of the figure. (b) Type-1 interferon genes and their receptors in Th17 cells differentiated with control or DSC conditioned medium, and in endometrial stromal cells. (c) 2'-5' Oligoadenylate synthase genes. (d) Eukaryotic translation initiation factors and some of their regulators. Red points in (c) and (d) represent genes significantly differentially expressed between control and DSC conditioned medium treated T cells according to EdgeR (see Methods). CPM = Counts per Million. 


\section{A model for the evolution of implantation}

386 Placing the results from this study in the context of previous studies, the following model for the evolution of embryo implantation emerges (Figure 7).
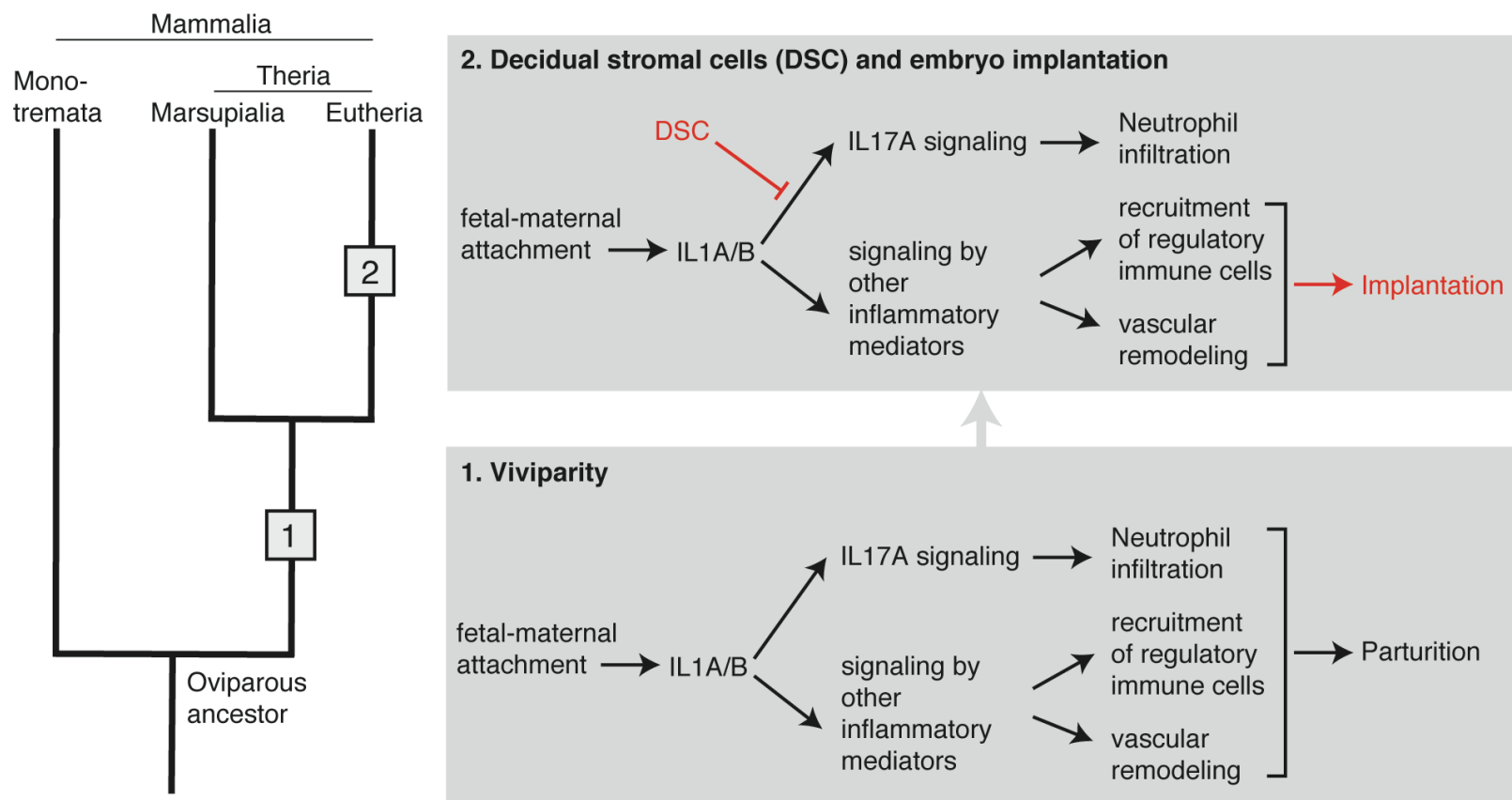

Figure 7 Model for the evolution of eutherian implantation from attachment-induced inflammation. Evolutionary events in the therian and eutherian stem lineages are represented by numbers 1 and 2 respectively.

The ancestor of all mammals was an egg-laying amniote. Mammalian viviparity originated in the stem lineage of therian mammals by early "hatching" of the embryo while it was still within the uterus/shell gland, leading to a direct physical contact between fetal membranes and the uterine endometrial lining. This novel tissue interaction, and potentially an irritation of the endometrial lining from fetal proteases that help dissolve the shell (Griffith, Chavan et al. 2017), induced an acute inflammatory response in the endometrium. An inflamed uterus, unable to maintain and nourish a live embryo within it, expelled the embryo, i.e. parturition ensued as a consequence of attachment-induced inflammation.

Hence we assume that the ancestral condition for therians was a typical mucosal inflammation in response to embryo attachment. However, in the stem lineage of eutherian mammals, this inflammatory response became evolutionarily modified such that the endometrium could maintain and nourish the embryo, even though it initiated an inflammation-like response. One of these modifications was the suppression of IL17A signaling, which resulted in the prevention of recruitment of neutrophils to the endometrium during implantation. This may have been a crucial modification to the inflammatory response since neutrophils are known to cause collateral tissue damage from the digestive enzymes they secrete; and the attaching embryo also would likely have not been spared from this tissue damage. Turning off IL17A early in the evolution of the 
eutherian lineage may have allowed the endometrium to maintain the embryo for a prolonged the maintenance and nourishment of the embryo, e.g. increased vascular permeability and angiogenesis may have helped nutrient transfer, therefore still maintained as a necessary part of implantation. The ancestral attachment-induced inflammation induced a stress reaction in the

414 endometrial stromal fibroblasts (ESF), causing the death of most of these cells. In the eutherian

415 lineage, however, ESF evaded the stress-induced cell death (Kajihara, Jones et al. 2006, Leitao,

416 Jones et al. 2010, Muter and Brosens 2018) by evolving mechanisms to differentiate into a novel 417 cell type, decidual stromal cells (DSC) (Erkenbrack, Maziarz et al. 2018). It is this novel cell 418 type that likely brought about the suppression of IL17A by inhibiting the differentiation of 419 IL17A-producing cells at the fetal-maternal interface, and thus enabled the evolution of embryo implantation and a sustainable fetal-maternal interface.

\section{Methods}

\section{Animals and tissue samples}

Nine-banded armadillos (Dasypus novemcinctus) were collected in Centerville, TX, USA, in accordance with Yale University IACUC protocol \#2014-10906. Two females were used in this study - one non-pregnant and one at the peri-implantation stage of pregnancy where the fetal membranes have begun invading the endometrium but no placental villi are yet formed. Rock hyrax (Procavia capensis) samples were collected at Bar-Ilan University, Israel. One out of three females collected was in the implantation phase of pregnancy as determined by histological examination. The blastocyst was attached to the uterine lumen but had not started invading the endometrium. Opossum (Monodelphis domestica) tissues were collected from the colony housed at Yale University. Samples were fixed in 4\% paraformaldehyde in phosphate-buffered saline (PBS) for histology and immunohistochemistry, saved in RNAlater (Ambion) for RNA extraction. For more information about armadillo samples, see (Chavan and Wagner 2016), and about opossum samples see (Griffith, Chavan et al. 2017). A list of animals used in this study is given in Supp. Table 1.

\section{Immunohistochemistry}

437 Formaldehyde-fixed tissues were dehydrated in ethanol, cleared in toluene, and embedded in 438 paraffin. Sections of $5 \mu \mathrm{m}$ thickness were made on a microtome and placed on poly-L-lysine coated glass slides. Immunohistochemistry was performed by following the protocol in (Chavan and Wagner 2016). Briefly, slides were incubated at $60^{\circ} \mathrm{C}$ for $30 \mathrm{~min}$ and allowed to cool at room temperature for $5 \mathrm{~min}$. Paraffin was removed by de-waxing the slides in xylene. Slides

442 were then rehydrated by successive washes in $100 \%$ ethanol, followed by running tap water.

443 Sodium Citrate buffer ( $\mathrm{pH}$ 6.0) was used for heat mediated antigen retrieval. After washing the 444 slides in PBS, they were blocked in a $0.1 \%$ solution of Bovine Serun Albumin (BSA) in PBS. 
445 Endogenous peroxidases were suppressed with Peroxidase Block (DAKO). Optimized dilutions

446 of primary antibodies (see Supp. Table 2) were added to the slides and were incubated overnight

447 at $4^{\circ} \mathrm{C}$ in a humidification chamber. Secondary antibody was added after washing the primary

448 antibody off with PBS and $0.1 \%$ BSA-PBS, and incubated for 1 hour at room temperature, and

449 washed off with PBS and 0.1\% BSA-PBS. Horseradish peroxidase (HRP) tagged secondary

450 antibodies were detected by either 3,3'-diaminobenzidine (DAB) and counterstained with

451 hematoxylin.

\section{Cell culture experiments}

453 DSC and DSC conditioned medium

454 Immortalized human endometrial stromal fibroblasts (hESF) from ATCC (ATCC; cat. no. CRL-

455 4003) were cultured in growth medium with the following contents per liter: $15.56 \mathrm{~g}$ DMEM

456 (D2906, Sigma-Aldrich), $1.2 \mathrm{~g}$ sodium bicarbonate, $10 \mathrm{ml}$ sodium pyruvate (11360, Thermo

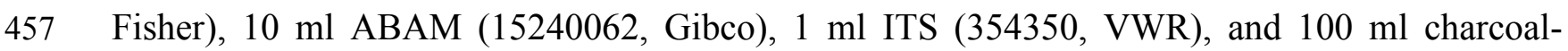

458 stripped FBS. ESF were differentiated into decidual stromal cells (DSC) in differentiation

459 medium with the following contents per liter: 15.56g DMEM (D8900, Sigma-Aldrich), 1.2g

460 sodium bicarbonate, $10 \mathrm{ml}$ ABAM, $0.5 \mathrm{mM}$ cAMP analog 8-Bromoadenosine 3',5'-cyclic

461 monophosphate sodium salt (B7880, Sigma-Aldrich), $1 \mu \mathrm{M}$ progesterone analog

462 Medroxyprogesterone 17-acetate (MPA; M1629, Sigma-Aldrich), and $20 \mathrm{ml} \mathrm{FBS.}$

463 Cells were differentiated for 48 hours at $37^{\circ} \mathrm{C}$, conditioned medium was collected from at least 6 464 replicate flasks, filtered through sterile $0.45 \mu \mathrm{m}$ filter to remove cell debris, aliquoted, and frozen 465 at $-80{ }^{\circ} \mathrm{C}$ until used. For control samples, decidualization medium was incubated for 48 hours at $46637^{\circ} \mathrm{C}$ without any cells in at least 6 replicate flasks, processed in the same way as conditioned 467 medium, and frozen at $-80{ }^{\circ} \mathrm{C}$.

468 T cells

469 Human Peripheral Blood Mononuclear Cells (PBMC) were isolated from whole blood (70501, 470 StemCell Technologies) using Lymphoprep (07851, StemCell Technologies) and $50 \mathrm{ml}$ SepMate 471 tubes (85450, StemCell Technologies). These PBMCs were used to isolate CD4+ CD45RO472 naïve $\mathrm{T}$ cells with EasySep Human Naïve CD4+ T Cell Isolation Kit (19555, StemCell 473 Technologies). Naïve $\mathrm{T}$ cells were resuspended at $10^{6}$ cells $/ \mathrm{ml}$ in ImmunoCult-XF $\mathrm{T}$ Cell 474 Expansion medium (10981, StemCell Technologies) in the presence of ImmunoCult 475 CD3/CD28/CD2 T Cell Activator (10970, StemCell Technologies) for culture or Th17 476 differentiation. For Th17 differentiation the following were added to the above cell suspension: $47720 \mathrm{ng} / \mathrm{ml}$ IL6 (78050, StemCell Technologies), 5 ng/ml TGFB1 (78067, StemCell Technologies), 478 and $50 \mathrm{ng} / \mathrm{ml}$ IL23 (14-8239-63, eBioScience). If conditioned medium was used, the above 479 suspension was made in 1:1 solution of conditioned or control medium and the T cell expansion 480 medium. Cell suspension was then transferred to 24 -well plates, with $10^{6}$ cells per well. Samples 481 with different treatments were placed in a Latin square design on 24-well plates to prevent 
482

483

484

485

486

487

488

489

490

491

492

493

494

495

496

497

498

499

500

501

502

503

504

505

506

507

508

509

510

511

512

513

514

515

516

517

systematic effects arising from the positions of the samples in the plate. Cells were incubated at $37^{\circ} \mathrm{C}$ for 7 days for differentiation. Cells, which are in suspension, were spun down to collect the supernatant and cell pellet. RNA was extracted from the cell pellets for RNA-seq (see below), and secreted IL17A was measured in the supernatants with Quantikine ELISA kit for human IL17A (D1700, R\&D Systems).

\section{RNA-seq data}

Among the animal tissue collected for this study, armadillo samples were used for RNAsequencing. RNA was extracted from whole uteri. Sequencing library for RNA from nonpregnant armadillo was prepared and sequenced at the Yale Center for Genome Analysis. Library preparation and sequencing of the RNA from implantation stage armadillo was performed at Cincinnati Children's Hospital Medical Center.

RNA from CD4+ naïve T cells differentiated into Th17 cells in the presence of DSC conditioned medium was extracted using Qiagen RNeasy Micro Kit (74004, Qiagen). Libraries were prepared and sequenced at the Yale Center for Genome Analysis.

Data for rabbit uterus (Oryctolagus cuniculus), grey short-tailed opossum (Monodelphis domestica) and human endometrial stromal cells were downloaded from Gene Expression Omnibus (GEO) (Barrett, Wilhite et al. 2013). GEO Accession numbers of the downloaded datasets as well as those generated in this study are listed in Supp. Table 3.

\section{RNA-seq analysis}

RNA-seq data were aligned to the following Ensembl genomes using TopHat2 (Kim, Pertea et al. 2013): GRCh37 for human, DasNov3 for armadillo, OryCun2 for rabbit, and MonDom5 for opossum. Number of reads mapping to genes were counted with HTSeq (Anders, Pyl et al. 2015). Read counts were normalized to Transcripts per Million (TPM) (Wagner, Kin et al. 2012), and 3 TPM was used as an operational threshold to call genes as expressed or unexpressed (Wagner, Kin et al. 2013). Median length of all transcripts of a gene was used as its 'feature length' for TPM normalization. Differential gene expression analysis for human $\mathrm{T}$ cell and endometrial stromal cell data was performed on protein coding genes with the $\mathrm{R}$ package EdgeR (Robinson, McCarthy et al. 2010). For these samples, read counts are normalized by EdgeR to Counts per Million (CPM), and are presented as such.

In analyses involving multiple species, only those genes were included that have one-to-one orthology among the species compared. Orthology data from Ensembl Compara database (Herrero, Muffato et al. 2016) were used. In these analyses, read counts were re-normalized to TPM using only the set of orthologous genes in order to make TPM values comparable between species.

Enriched gene ontology (GO) categories in sets of genes were identified with the online tool GOrilla (Eden, Navon et al. 2009). Lists of enriched GO categories were visualized on REViGO 
518 (Supek, Bošnjak et al. 2011), which clusters semantically similar GO terms in space, simplifying

519 interpretation of long lists of redundant GO categories.

520 qPCR

521 Expression of IL17A through opossum pregnancy was measured using qPCR at a finer time

522 scale than the trascriptomic data. RNA from non-pregnant, $8 \mathrm{dpc}, 11.5 \mathrm{dpc}, 12.5 \mathrm{dpc}$, and 13.5

523 dpc uteri was extracted and reverse transcribed to cDNA using High Capacity Reverse

524 Transcriptase Kit (4368814, Thermo Fisher). Abundance of IL17A mRNA was measured

525 relative to TBP (Tata Binding Protein) mRNA with a standard curve approach using Power

526 SYBR Green PCR Master Mix (4367659, Applied Biosystems) on StepOne Plus Real Time PCR

527 System (Applied Biosystems). Primer sequences are in Supp. Table 4.

\section{Acknowledgements}

529 This research was supported by John Templeton Foundation Grant \#54860 to GPW. We would 530 like to thank Haleigh Larson for assistance with histology.

\section{Competing Interests}

532 No competing interests to declare.

\section{References}

534 Aluvihare, V. R., M. Kallikourdis and A. G. Betz (2004). "Regulatory T cells mediate maternal 535 tolerance to the fetus." Nat Immunol 5(3): 266-271.

536 Anders, S., P. T. Pyl and W. Huber (2015). "HTSeq-a Python framework to work with high537 throughput sequencing data." Bioinformatics 31(2): 166-169.

538 Ashary, N., A. Tiwari and D. Modi (2018). "Embryo Implantation: War in Times of Love." 539 Endocrinology 159(2): 1188-1198.

540 Barash, A., N. Dekel, S. Fieldust, I. Segal, E. Schechtman and I. Granot (2003). "Local injury to 541 the endometrium doubles the incidence of successful pregnancies in patients undergoing in vitro 542 fertilization." Fertility and Sterility 79(6): 1317-1322.

543 Barrett, T., S. E. Wilhite, P. Ledoux, C. Evangelista, I. F. Kim, M. Tomashevsky, K. A. 544 Marshall, K. H. Phillippy, P. M. Sherman, M. Holko, A. Yefanov, H. Lee, N. Zhang, C. L. 545 Robertson, N. Serova, S. Davis and A. Soboleva (2013). "NCBI GEO: archive for functional 546 genomics data sets—update." Nucleic Acids Research 41(Database issue): D991-D995.

547 Bellofiore, N., S. J. Ellery, J. Mamrot, D. W. Walker, P. Temple-Smith and H. Dickinson (2017).

548 "First evidence of a menstruating rodent: the spiny mouse (Acomys cahirinus)." American

549 Journal of Obstetrics and Gynecology 216(1): 40.e41-40.e11. 
Bettelli, E., Y. Carrier, W. Gao, T. Korn, T. B. Strom, M. Oukka, H. L. Weiner and V. K. Kuchroo (2006). "Reciprocal developmental pathways for the generation of pathogenic effector TH17 and regulatory T cells." Nature 441: 235.

Buckley, K. M., E. C. H. Ho, T. Hibino, C. S. Schrankel, N. W. Schuh, G. Wang and J. P. Rast (2017). "IL17 factors are early regulators in the gut epithelium during inflammatory response to Vibrio in the sea urchin larva." eLife 6: e23481.

Chavan, A. R., B.-A. S. Bhullar and G. P. Wagner (2016). "What was the ancestral function of decidual stromal cells? A model for the evolution of eutherian pregnancy." Placenta 40: 40-51.

Chavan, A. R., O. W. Griffith and G. P. Wagner (2017). "The inflammation paradox in the evolution of mammalian pregnancy: turning a foe into a friend." Curr Opin Genet Dev 47: 24-32.

Chavan, A. R. and G. P. Wagner (2016). "The fetal-maternal interface of the nine-banded armadillo: endothelial cells of maternal sinus are partially replaced by trophoblast." Zoological Letters 2(1): 11 .

Chen, Q., Y. Zhang, J. Lu, Q. Wang, S. Wang, Y. Cao, H. Wang and E. Duan (2009). "Embryouterine cross-talk during implantation: the role of Wnt signaling $\dagger . "$ MHR: Basic science of reproductive medicine 15(4): 215-221.

Choi, G. B., Y. S. Yim, H. Wong, S. Kim, H. Kim, S. V. Kim, C. A. Hoeffer, D. R. Littman and J. R. Huh (2016). "The maternal interleukin-17a pathway in mice promotes autism-like phenotypes in offspring." Science 351(6276): 933.

Dekel, N., Y. Gnainsky, I. Granot, K. Racicot and G. Mor (2014). "The Role of Inflammation for a Successful Implantation." American Journal of Reproductive Immunology 72(2): 141-147.

dos Reis, M., J. Inoue, M. Hasegawa, R. J. Asher, P. C. Donoghue and Z. Yang (2012). "Phylogenomic datasets provide both precision and accuracy in estimating the timescale of placental mammal phylogeny." Proc Biol Sci 279(1742): 3491-3500.

Dufour, J. H., M. Dziejman, M. T. Liu, J. H. Leung, T. E. Lane and A. D. Luster (2002). "IFN- $\gamma-$ Inducible Protein 10 (IP-10; CXCL10)-Deficient Mice Reveal a Role for IP-10 in Effector T Cell Generation and Trafficking." The Journal of Immunology 168(7): 3195.

Eden, E., R. Navon, I. Steinfeld, D. Lipson and Z. Yakhini (2009). "GOrilla: a tool for discovery and visualization of enriched GO terms in ranked gene lists." BMC Bioinformatics 10(1): 48.

Emera, D., R. Romero and G. Wagner (2011). "The evolution of menstruation: A new model for genetic assimilation." BioEssays 34(1): 26-35.

Enders, A. C. and S. Schlafke (1969). "Cytological aspects of trophoblast-uterine interaction in early implantation." Am J Anat 125(1): 1-29. 
Erkenbrack, E. M., J. D. Maziarz, O. W. Griffith, C. Liang, A. R. Chavan, M. C. Nnamani and G. P. Wagner (2018). "The mammalian decidual cell evolved from a cellular stress response." PLOS Biology 16(8): e2005594.

Erlebacher, A. (2013). "Immunology of the maternal-fetal interface." Annu. Rev. Immunol. 31: 387-411.

Finn, C. A. (1986). "Implantation, menstruation and inflammation." Biological Reviews 61(4): 313-328.

Flannigan, K. L., V. L. Ngo, D. Geem, A. Harusato, S. A. Hirota, C. A. Parkos, N. W. Lukacs, A. Nusrat, V. Gaboriau-Routhiau, N. Cerf-Bensussan, A. T. Gewirtz and T. L. Denning (2016). "IL-17A-mediated neutrophil recruitment limits expansion of segmented filamentous bacteria." Mucosal Immunology 10: 673.

Gellersen, B. and J. J. Brosens (2014). "Cyclic decidualization of the human endometrium in reproductive health and failure." Endocr Rev 35(6): 851-905.

Griffin, G. K., G. Newton, M. L. Tarrio, D.-x. Bu, E. Maganto-Garcia, V. Azcutia, P. Alcaide, N. Grabie, F. W. Luscinskas, K. J. Croce and A. H. Lichtman (2012). "IL-17 and TNF- $\alpha$ Sustain Neutrophil Recruitment during Inflammation through Synergistic Effects on Endothelial Activation." The Journal of Immunology 188(12): 6287.

Griffith, O. W., A. R. Chavan, S. Protopapas, J. Maziarz, R. Romero and G. P. Wagner (2017). "Embryo implantation evolved from an ancestral inflammatory attachment reaction." Proceedings of the National Academy of Sciences doi: 10.1073/pnas.1701129114.

Griffith, O. W., A. R. Chavan, S. Protopapas, J. Maziarz, R. Romero and G. P. Wagner (2018). "Reply to Liu: Inflammation before implantation both in evolution and development." Proc Natl Acad Sci U S A 115(1): E3-E4.

Hansen, V. L., L. S. Faber, A. A. Salehpoor and R. D. Miller (2017). "A pronounced uterine proinflammatory response at parturition is an ancient feature in mammals." Proceedings of the Royal Society B: Biological Sciences 284(1865).

Herrero, J., M. Muffato, K. Beal, S. Fitzgerald, L. Gordon, M. Pignatelli, A. J. Vilella, S. M. J. Searle, R. Amode, S. Brent, W. Spooner, E. Kulesha, A. Yates and P. Flicek (2016). "Ensembl comparative genomics resources." Database 2016: bav096-bav096.

Kajihara, T., M. Jones, L. Fusi, M. Takano, F. Feroze-Zaidi, G. Pirianov, H. Mehmet, O. Ishihara, J. M. Higham, E. W. Lam and J. J. Brosens (2006). "Differential expression of FOXO1 and FOXO3a confers resistance to oxidative cell death upon endometrial decidualization." Mol Endocrinol 20(10): 2444-2455.

Keys, J. L., G. J. King and T. G. Kennedy (1986). "Increased uterine vascular permeability at the time of embryonic attachment in the pig." Biology of Reproduction 34(2): 405-411. 
Kim, D., G. Pertea, C. Trapnell, H. Pimentel, R. Kelley and S. L. Salzberg (2013). "TopHat2: accurate alignment of transcriptomes in the presence of insertions, deletions and gene fusions." Genome Biology 14(4): R36.

Kin, K., J. Maziarz, A. R. Chavan, M. Kamat, S. Vasudevan, A. Birt, D. Emera, V. J. Lynch, T. L. Ott, M. Pavlicev and G. P. Wagner (2016). "The Transcriptomic Evolution of Mammalian Pregnancy: Gene Expression Innovations in Endometrial Stromal Fibroblasts." Genome Biol Evol 8(8): 2459-2473.

Kindt, T. J., R. A. Goldsby and B. A. Osborne (2007). Kuby immunology. New York, W.H. Freeman and Company.

Leitao, B., M. C. Jones, L. Fusi, J. Higham, Y. Lee, M. Takano, T. Goto, M. Christian, E. W. F. Lam and J. J. Brosens (2010). "Silencing of the JNK pathway maintains progesterone receptor activity in decidualizing human endometrial stromal cells exposed to oxidative stress signals." Faseb j 24(5): 1541-1551.

Li, N., Q. Qu and Q. Yan (2018). "The role of Th17/Treg-mediated immunoregulation in abortion mice." European Journal of Inflammation 16: 2058739218760354.

Lieschke, G. J., D. Grail, G. Hodgson, D. Metcalf, E. Stanley, C. Cheers, K. J. Fowler, S. Basu, Y. F. Zhan and A. R. Dunn (1994). "Mice lacking granulocyte colony-stimulating factor have chronic neutropenia, granulocyte and macrophage progenitor cell deficiency, and impaired neutrophil mobilization." Blood 84(6): 1737.

Liu, J.-L. (2018). "Implantation in eutherians: Which came first, the inflammatory reaction or attachment?" Proceedings of the National Academy of Sciences 115(1): E1.

Liu, J.-L., M. Zhao, Y. Peng and Y.-S. Fu (2016). "Identification of gene expression changes in rabbit uterus during embryo implantation." Genomics 107(5): 216-221.

Marions, L. and K. G. Danielsson (1999). "Expression of cyclo-oxygenase in human endometrium during the implantation period." Mol Hum Reprod 5(10): 961-965.

McAllan, B. M. (2011). Chapter 10 - Reproductive Endocrinology of Prototherians and Metatherians. Hormones and Reproduction of Vertebrates. D. O. N. H. Lopez. London, Academic Press: 195-214.

Medzhitov, R. (2007). "Recognition of microorganisms and activation of the immune response." Nature 449: 819.

Mess, A. and A. M. Carter (2006). "Evolutionary transformations of fetal membrane characters in Eutheria with special reference to Afrotheria." J Exp Zool B Mol Dev Evol 306(2): 140-163.

Milne, S. A., G. B. Perchick, S. C. Boddy and H. N. Jabbour (2001). "Expression, localization, and signaling of PGE(2) and EP2/EP4 receptors in human nonpregnant endometrium across the menstrual cycle." J Clin Endocrinol Metab 86(9): 4453-4459. 
Mohamed, O. A., M. Jonnaert, C. Labelle-Dumais, K. Kuroda, H. J. Clarke and D. Dufort (2005). "Uterine Wnt/ $\beta$-catenin signaling is required for implantation." Proceedings of the National Academy of Sciences of the United States of America 102(24): 8579.

Mor, G., I. Cardenas, V. Abrahams and S. Guller (2011). "Inflammation and pregnancy: the role of the immune system at the implantation site." Annals of the New York Academy of Sciences 1221(1): 80-87.

Mossman, H. W. (1937). "Comparative morphogenesis of the fetal membranes and accessory uterine structures." Contributions to Embryology 26(158): 133-137.

Muter, J. and J. J. Brosens (2018). Decidua. Encyclopedia of Reproduction (Second Edition). M. K. Skinner. Oxford, Academic Press: 424-430.

Nakashima, A., M. Ito, T. Shima, D. Bac Nguyen, T. Hidaka and S. Saito (2010). "Accumulation of IL - 17 - Positive Cells in Decidua of Inevitable Abortion Cases." American Journal of Reproductive Immunology 64(1): 4-11.

Nancy, P., E. Tagliani, C. S. Tay, P. Asp, D. E. Levy and A. Erlebacher (2012). "Chemokine Gene Silencing in Decidual Stromal Cells Limits T Cell Access to the Maternal-Fetal Interface." Science 336(6086): 1317-1321.

Onishi, R. M. and S. L. Gaffen (2010). "Interleukin-17 and its target genes: mechanisms of interleukin-17 function in disease." Immunology 129(3): 311-321.

Panopoulos, A. D. and S. S. Watowich (2008). "Granulocyte Colony-Stimulating Factor: molecular mechanisms of action during steady state and 'emergency' hematopoiesis." Cytokine 42(3): 277-288.

Pappu, R., S. Rutz and W. Ouyang (2012). "Regulation of epithelial immunity by IL-17 family cytokines." Trends in Immunology 33(7): 343-349.

Pavlicev, M., G. P. Wagner, A. R. Chavan, K. Owens, J. Maziarz, C. Dunn-Fletcher, S. G. Kallapur, L. Muglia and H. Jones (2017). "Single-cell transcriptomics of the human placenta: inferring the cell communication network of the maternal-fetal interface." Genome Res 27(3): 349-361.

Pinget, G. V., T. M. Corpuz, J. Stolp, E. L. Lousberg, K. R. Diener, S. A. Robertson, J. Sprent and K. E. Webster (2016). "The majority of murine $\gamma \delta \mathrm{T}$ cells at the maternal-fetal interface in pregnancy produce IL-17." Immunology And Cell Biology 94: 623.

Plaks, V., T. Birnberg, T. Berkutzki, S. Sela, A. BenYashar, V. Kalchenko, G. Mor, E. Keshet, N. Dekel, M. Neeman and S. Jung (2008). "Uterine DCs are crucial for decidua formation during embryo implantation in mice." The Journal of Clinical Investigation 118(12): 3954-3965.

Renfree, M. (1994). Endocrinology of Pregnancy, Parturition and Lactation in Marsupials. Marshall's Physiology of Reproduction. G. E. Lamming, Springer Netherlands: 677-766. 
Robertson, S. A. and L. M. Moldenhauer (2014). "Immunological determinants of implantation success." Int J Dev Biol 58(2-4): 205-217.

Robinson, M. D., D. J. McCarthy and G. K. Smyth (2010). "edgeR: a Bioconductor package for differential expression analysis of digital gene expression data." Bioinformatics 26(1): 139-140.

Schall, T. J. (1991). "Biology of the rantes/sis cytokine family." Cytokine 3(3): 165-183.

Schlafke, S. and A. C. Enders (1975). "Cellular basis of interaction between trophoblast and uterus at implantation." Biol Reprod 12(1): 41-65.

Selwood, L. (2000). "Marsupial egg and embryo coats." Cells Tissues Organs 166(2): 208-219.

Shuya, L. L., E. M. Menkhorst, J. Yap, P. Li, N. Lane and E. Dimitriadis (2011). "Leukemia Inhibitory Factor enhances endometrial stromal cell decidualization in humans and mice." PLOS ONE 6(9): e25288.

Sonderegger, S., J. Pollheimer and M. Knöfler (2010). "Wnt Signalling in Implantation, Decidualisation and Placental Differentiation -- review." Placenta 31(10): 839-847.

Supek, F., M. Bošnjak, N. Škunca and T. Šmuc (2011). "REVIGO Summarizes and Visualizes Long Lists of Gene Ontology Terms." PLOS ONE 6(7): e21800.

Talukder, A. K., M. B. Rashid, M. S. Yousef, K. Kusama, T. Shimizu, M. Shimada, S. S. Suarez, K. Imakawa and A. Miyamoto (2018). "Oviduct epithelium induces interferon-tau in bovine Day-4 embryos, which generates an anti-inflammatory response in immune cells." Scientific Reports 8(1): 7850 .

Tarver, J. E., M. dos Reis, S. Mirarab, R. J. Moran, S. Parker, J. E. O’Reilly, B. L. King, M. J. O’Connell, R. J. Asher, T. Warnow, K. J. Peterson, P. C. J. Donoghue and D. Pisani (2016). "The Interrelationships of Placental Mammals and the Limits of Phylogenetic Inference." Genome Biology and Evolution.

Van Sinderen, M., E. Menkhorst, A. Winship, C. Cuman and E. Dimitriadis (2013).

"Preimplantation Human Blastocyst-Endometrial Interactions: The Role of Inflammatory Mediators." American Journal of Reproductive Immunology 69(5): 427-440.

Waclawik, A. and A. J. Ziecik (2007). "Differential expression of prostaglandin (PG) synthesis enzymes in conceptus during peri-implantation period and endometrial expression of carbonyl reductase/PG 9-ketoreductase in the pig." Journal of Endocrinology 194(3): 499-510.

Wagner, G. P., K. Kin and V. J. Lynch (2012). "Measurement of mRNA abundance using RNAseq data: RPKM measure is inconsistent among samples." Theory Biosci 131(4): 281-285.

Wagner, G. P., K. Kin and V. J. Lynch (2013). "A model based criterion for gene expression calls using RNA-seq data." Theory Biosci 132(3): 159-164. 
Wagner, G. P., K. Kin, L. Muglia and M. Pavlicev (2014). "Evolution of mammalian pregnancy and the origin of the decidual stromal cell." Int J Dev Biol 58(2-4): 117-126.

Wang, H. and S. K. Dey (2006). "Roadmap to embryo implantation: clues from mouse models."

724 Nature Reviews Genetics 7: 185.

Wang, W.-J., C.-F. Hao, L. Yi, G.-J. Yin, S.-H. Bao, L.-H. Qiu and Q.-D. Lin (2010). "Increased prevalence of T helper 17 (Th17) cells in peripheral blood and decidua in unexplained recurrent spontaneous abortion patients." Journal of Reproductive Immunology 84(2): 164-170.

Whittington, C. M., D. O’Meally, M. K. Laird, K. Belov, M. B. Thompson and B. M. McAllan (2018). "Transcriptomic changes in the pre-implantation uterus highlight histotrophic nutrition of the developing marsupial embryo." Scientific Reports 8(1): 2412.

Whyte, J. J., A. E. Meyer, L. D. Spate, J. A. Benne, R. Cecil, M. S. Samuel, C. N. Murphy, R. S. Prather and R. D. Geisert (2017). "Inactivation of porcine interleukin-1 $\beta$ results in failure of rapid conceptus elongation." Proceedings of the National Academy of Sciences.

Wu, H.-X., L.-P. Jin, B. Xu, S.-S. Liang and D.-J. Li (2014). "Decidual stromal cells recruit Th17 cells into decidua to promote proliferation and invasion of human trophoblast cells by secreting IL-17." Cellular And Molecular Immunology 11: 253.

Ye, P., F. H. Rodriguez, S. Kanaly, K. L. Stocking, J. Schurr, P. Schwarzenberger, P. Oliver, W. Huang, P. Zhang, J. Zhang, J. E. Shellito, G. J. Bagby, S. Nelson, K. Charrier, J. J. Peschon and J. K. Kolls (2001). "Requirement of Interleukin 17 Receptor Signaling for Lung Cxc Chemokine and Granulocyte Colony-Stimulating Factor Expression, Neutrophil Recruitment, and Host Defense." The Journal of Experimental Medicine 194(4): 519.

Yockey, L. J. and A. Iwasaki (2018). "Interferons and Proinflammatory Cytokines in Pregnancy and Fetal Development." Immunity 49(3): 397-412.

Yockey, L. J., K. A. Jurado, N. Arora, A. Millet, T. Rakib, K. M. Milano, A. K. Hastings, E. Fikrig, Y. Kong, T. L. Horvath, S. Weatherbee, H. J. Kliman, C. B. Coyne and A. Iwasaki (2018). "Type I interferons instigate fetal demise after Zika virus infection." Science Immunology 3(19). 